\title{
Competition of Species with Intra-Specific Competition
}

\author{
N. Apreutesei ${ }^{a 1}$, A. Ducrot ${ }^{b}$ and V. Volpert ${ }^{c}$ \\ ${ }^{a}$ Department of Mathematics, Technical University of Iasi, Iasi, Romania \\ ${ }^{b}$ Institute of Mathematics, University Bordeaux 2, 33076 Bordeaux, France \\ ${ }^{c}$ Institute of Mathematics, Université Lyon 1, UMR 5208 CNRS, 69622 Villeurbanne, France
}

\begin{abstract}
Intra-specific competition in population dynamics can be described by integro-differential equations where the integral term corresponds to nonlocal consumption of resources by individuals of the same population. Already the single integro-differential equation can show the emergence of nonhomogeneous in space stationary structures and can be used to model the process of speciation, in particular, the emergence of biological species during evolution [6], [7]. On the other hand, competition of two different species represents a well known and well studied model in population dynamics. In this work we study how the intra-specific competition can influence the competition between species. We will prove the existence of travelling waves for the case where the support of the kernel of the integral is sufficiently narrow. Numerical simulations will be carried out in the case of large supports.
\end{abstract}

Key words: integro-differential equation, Fredholm property, travelling waves

AMS subject classification: 35K57, 35R10, 47A53, 92D25

\section{Models in population dynamics with intra-specific competi- tion}

Importance of intra-specific competition for various questions in population dynamics is accepted already long time ago due to Darwin's famous book on the origin of species. One of the simplest models taking into account intra-specific competition is given by the integro-differential equation

$$
\frac{\partial u}{\partial t}=d \frac{\partial^{2} u}{\partial x^{2}}+k u\left(1-\int_{-\infty}^{\infty} \varphi(x-y) u(y, t) d y\right) .
$$

\footnotetext{
${ }^{1}$ Corresponding author. E-mail: napreut@net89mail.dntis.ro
} 
Here $u$ is the density of a population, the first term in the right-hand side describes displacement of the individuals either in the physical space or in the morphological space. In the latter case, $x$ corresponds to some morphological characteristics, for example, the weight of some animals, their height, or some other metrical characterisics. The second term in the right-hand side describes the reproduction of the population which is proportional to its density and to available resources, the expression in the brackets. If $\varphi(y)$ is the Dirac $\delta$-function, then the integral becomes equal to $u(x, t)$, and we obtain one of the classical models in population dynamics, the reaction-diffusion equation

$$
\frac{\partial u}{\partial t}=d \frac{\partial^{2} u}{\partial x^{2}}+F(u)
$$

where $F(u)=k u(1-u)$. If $\varphi(y)$ has a finite support, then the integral describes the consumption of resources at the space point $x$ by the individuals located at the space point $y$. Thus, we deal with nonlocal consumption of resources or, in the other words, with the intra-specific competition, that is the competition of individuals of the same species for resources.

Reaction-diffusion equation (1.2) was first studied in the context of population dynamics by Fisher [5] and Kolmogorov, Petrovskii, Piskunov [9], and then in a big number of works for various nonlinearities $F(u)$ (see [13] and the references therein). The main results concern the existence and stability of travelling waves, that is solutions of the form $u(x, t)=w(x-c t)$, where $c$ is the wave speed.

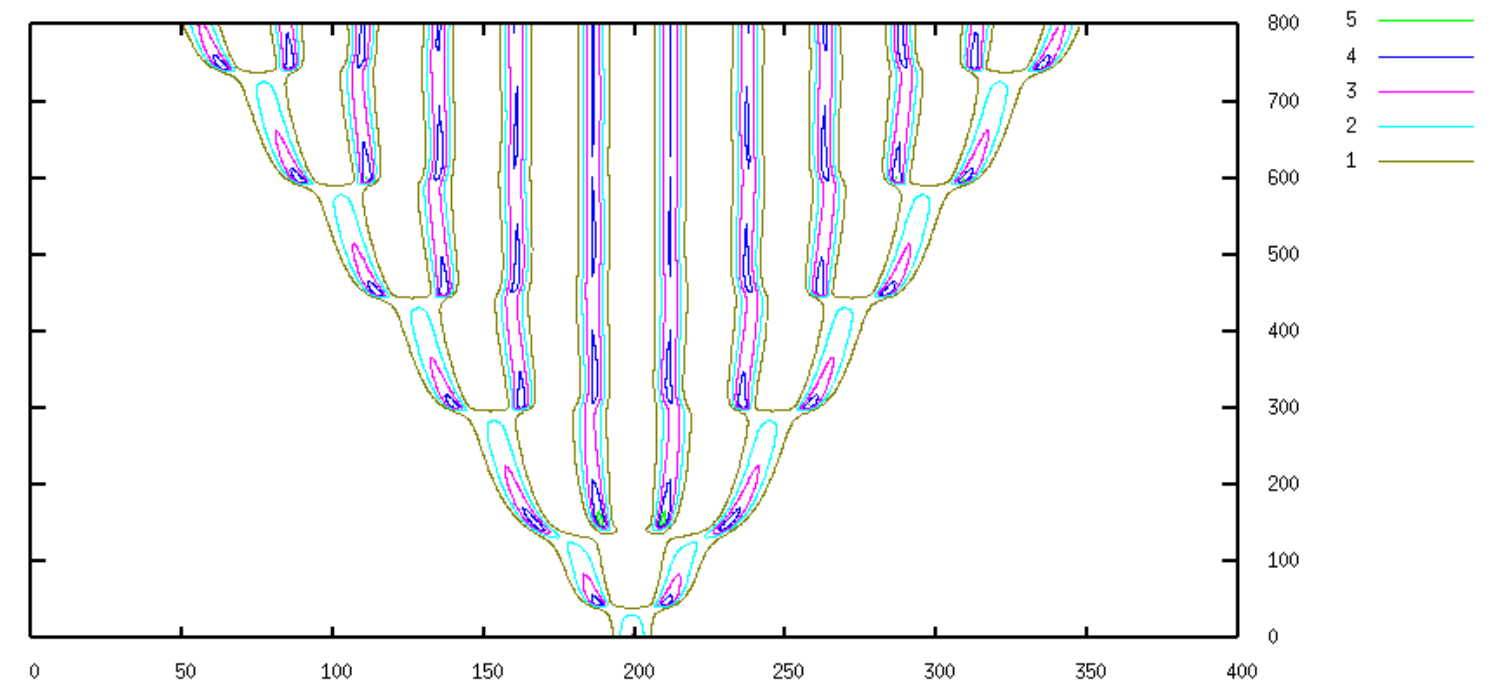

Figure 1: Emergence of new species: level lines of the density in the bistable case.

The wave existence can be proved if the support of $\varphi$ is sufficiently narrow. For the monostable case (1.1) it can be done by constructing some approximating sequences (see [4] and the references therein). In the bistable case,

$$
\frac{\partial u}{\partial t}=d \frac{\partial^{2} u}{\partial x^{2}}+k u^{2}\left(1-\int_{-\infty}^{\infty} \varphi(x-y) u(y, t) d y\right)-b u
$$


which describes bisexual reproduction, it is proved in our previous work [1] by the implicit function theorem.

In both cases the waves have the limits $u=0$ and $u=1$ at infinity. If the support of $\varphi$ becomes sufficiently large, then the homogeneous in space solution $u=1$ loses its stability, and a periodic in space stationary solution emerges [6], [7], [8]. It is possible that the wave with the limit $u(-\infty)=1$ still exists but becomes unstable. Numerical simulations show propagation of periodic waves. An example of such simulations is shown in Figure 1 ([1]). It represents level lines of the solution $u(x, t)$ of equation (1.3). The horizontal axis is the $x$-variable, the vertical axis is time. The initial condition is localized in a narrow interval near $x=200$. We see that a periodic in space structure emerges at the center of the interval and propagates to the left and to the right in the form of periodic travelling waves.

There are various biological interpretations of these results. One of the most interesting is related to the evolution of biological species. If we consider the space variable $x$ as a morphological parameter, then the individuals that have approximately the same value of this parameter can be interpreted as a biological species, while two populations separated in the morphological space correspond to two different species. In the simulations shown in Figure 1, initially we have a unique species with approximately the same value of the morphological parameter. After some time, it splits into two subpopulations or two different species. Some time later, new species appear and so on until the whole morphological space is completely filled. These results correspond to Darwin's description of the emergence of biological species in the process of evolution. In our modelling, it is based on three properties: random mutations (diffusion), intra-specific competition (integral), self-reproduction with the same phenotype (nonlinear term). It is interesting to note that the process of speciation can be modelled in a similar way for many other applications including the production of consumer goods or specialization in art and in science.

Thus, intra-specific competition can result in the emergence of new biological species. The new species continue to compete for resources in both ways, intra-specifically and between the species. Competition of species is a classical problem in population dynamics which can be described in the simplest case by the reaction-diffusion system:

$$
\begin{aligned}
& \frac{\partial u}{\partial t}=d_{1} \frac{\partial^{2} u}{\partial x^{2}}+F(u, v), \\
& \frac{\partial v}{\partial t}=d_{2} \frac{\partial^{2} v}{\partial x^{2}}+G(u, v)
\end{aligned}
$$

where

$$
F(u, v)=k_{1} u\left(1-a_{1} u-b_{1} v\right)-p_{1} u, \quad G(u, v)=k_{2} v\left(1-a_{2} u-b_{2} v\right)-p_{2} v .
$$

For each function $F$ and $G$, the first expression in the right-hand side describes natality, which is proportional to the density of the population and to available resources (expression in the brackets), the second term describes mortality. Depending on the parameters, there are two basic situations: coexistence of the two species and disappearance of one species while another one spreads and occupies the whole space. The ordinary differential system of equations

$$
\frac{d u}{d t}=F(u, v), \quad \frac{d v}{d t}=G(u, v)
$$


can have four stationary points:

$$
P_{0}=(0,0), \quad P_{1}=\left(\frac{k_{1}-p_{1}}{k_{1} a_{1}}, 0\right) \quad P_{2}=\left(0, \frac{k_{2}-p_{2}}{k_{2} b_{2}}\right), \quad P_{4}=\left(a_{*}, b_{*}\right),
$$

where $\left(a_{*}, b_{*}\right)$ is a solution of the system

$$
a_{1} u+b_{1} v=1-\frac{p_{1}}{k_{1}}, \quad a_{2} u+b_{2} v=1-\frac{p_{2}}{k_{2}}
$$

if such solution exists and if it is non-negative. We will suppose that $a_{*}$ and $b_{*}$ are strictly positive.

If the point $P_{4}$ is stable with respect to system (1.7), then this is the case of co-existence of species. Solution of reaction-diffusion system (1.4), (1.5) considered on the whole axis with properly chosen initial conditions converges to $P_{4}$ on every bounded space interval. If this point is instable, then the points $P_{1}$ and $P_{2}$ are stable. In this case there exists a travelling wave solution $u(x, t)=w_{1}(x-c t), v(x, t)=w_{2}(x-c t)$ of this system with the limits $P_{1}$ and $P_{2}$ at infinity. If the speed $c$ of this wave is different from zero, then one of the two species invades the whole space, while another one disappears. If $c=0$, then the species co-exist but they are separated in space except for some transition zone.

In this work we study how intra-specific competition can influence the competition between two different species. In order to take into account intra-specific competition we replace $F$ and $G$ in (1.4), (1.5) by the expressions

$$
\begin{aligned}
& F:=k_{1} u^{m}\left(1-a_{1} \int_{-\infty}^{\infty} \varphi(x-y) u(y, t) d y-b_{1} \int_{-\infty}^{\infty} \varphi(x-y) v(y, t) d y\right)-p_{1} u, \\
& G:=k_{2} v^{m}\left(1-a_{2} \int_{-\infty}^{\infty} \varphi(x-y) u(y, t) d y-b_{2} \int_{-\infty}^{\infty} \varphi(x-y) v(y, t) d y\right)-p_{2} v .
\end{aligned}
$$

In the case where the function $\varphi$ has a sufficiently narrow support, we will study the existence of travelling waves connecting stable stationary points $P_{1}$ and $P_{2}$ (Section 3 ). If we replace formally $\varphi$ by the Dirac $\delta$-function, we obtain the reaction-diffusion system (1.4), (1.5) for which the existence of waves is known. Therefore, we can expect that they also exist for functions $\varphi$ with narrow supports. In order to prove this result, we need to study properties of the corresponding integrodifferential operators (Section 2).

In Section 4 we discuss the result of numerical simulations both for narrow and large support of $\varphi$. In the former case the behavior of solutions is similar to that for the reaction-diffusion system. In the latter, the homogeneous in space solutions can lose their stability. Instead of the usual travelling waves, we will observe propagation of periodic waves. Some biological interpretations of the results are discussed in Section 5. 


\section{Properties of integro-differential operators}

We are concerned with the system of integro-differential equations

$$
\left\{\begin{aligned}
\frac{\partial u_{1}}{\partial t} & =\alpha_{1} \frac{\partial^{2} u_{1}}{\partial x^{2}}+F_{1}\left(u_{1}, \ldots, u_{n}, \varphi_{11} * u_{1}, \ldots, \varphi_{1 n} * u_{n}\right) \\
\frac{\partial u_{n}}{\partial t} & =\alpha_{n} \frac{\partial^{2} u_{n}}{\partial x^{2}}+F_{n}\left(u_{1}, \ldots, u_{n}, \varphi_{n 1} * u_{1}, \ldots, \varphi_{n n} * u_{n}\right)
\end{aligned}\right.
$$

where $\alpha_{i}>0, i=1, \ldots, n$ are given constants, $\varphi_{i j}, i, j=1, \ldots, n$ are functions from $L^{1}(\mathbb{R})$, such that $\int_{-\infty}^{\infty} \varphi_{i j}(y) d y=1$, and $F_{1}, \ldots, F_{n}: \mathbb{R}^{2 n} \rightarrow \mathbb{R}$ are given functions with some specific properties. By $\varphi_{i j} * u_{j}$ we mean the convolution product

$$
\left(\varphi_{i j} * u_{j}\right)(x)=\int_{-\infty}^{\infty} \varphi_{i j}(x-y) u_{j}(y) d y .
$$

Let $u=\left(u_{1}, \ldots, u_{n}\right)^{T}, F=\left(F_{1}, \ldots, F_{n}\right)^{T}$, where the superscript $T$ denotes the transposed vector. Assume that $F \in C^{1}\left(\mathbb{R}^{2 n}, \mathbb{R}^{n}\right)$.

Observe that if the functions $\varphi_{i j}$ have bounded supports $\left[-N_{i j}, N_{i j}\right]$ with $N_{i j} \rightarrow 0$, then the above system of integro-differential equations becomes in the limit, at least formally, the reactiondiffusion system

$$
\frac{\partial u_{i}}{\partial t}=\alpha_{i} \frac{\partial^{2} u_{i}}{\partial x^{2}}+F_{i}(u, u), \quad i=1, \ldots, n .
$$

Travelling wave solutions of system (2.1) are solutions of the form $u(x, t)=w(x-c t)$, where $c$ is the wave speed. If $w=\left(w_{1}, \ldots, w_{n}\right)^{T}$, then $u_{i}(x, t)=w_{i}(x-c t), i=1, \ldots, n$ and the functions $w_{i}$ satisfy the equations

$$
\alpha_{i} w_{i}^{\prime \prime}+c w_{i}^{\prime}+F_{i}\left(w_{1}, \ldots, w_{n}, \varphi_{i 1} * w_{1}, \ldots, \varphi_{i n} * w_{n}\right)=0, i=1, \ldots, n .
$$

Let $A_{i}:\left(C^{2+\alpha}(\mathbb{R})\right)^{n} \rightarrow C^{\alpha}(\mathbb{R}), i=1, \ldots, n$, be the operator corresponding to the left-hand side of $(2.2)$, that is

$$
A_{i} u=\alpha_{i} u_{i}^{\prime \prime}+c u_{i}^{\prime}+F_{i}\left(u_{1}, \ldots, u_{n}, \varphi_{i 1} * u_{1}, \ldots, \varphi_{i n} * u_{n}\right), i=1, \ldots, n
$$

and $A:\left(C^{2+\alpha}(\mathbb{R})\right)^{n} \rightarrow\left(C^{\alpha}(\mathbb{R})\right)^{n}$ be given by $A u=\left(A_{1} u, \ldots, A_{n} u\right)$. Denote by $B$ the diagonal matrix with the elements $\alpha_{i}, i=1, \ldots, n$ at the principal diagonal and by $\Phi * u$ the matrix

$$
\Phi * u=\left(\begin{array}{ccc}
\varphi_{11} * u_{1} & \ldots & \varphi_{1 n} * u_{n} \\
\ldots & \ldots & \ldots \\
\varphi_{n 1} * u_{1} & \ldots & \varphi_{n n} * u_{n}
\end{array}\right)
$$

Then $A u=B u^{\prime \prime}+c u^{\prime}+F(u, \Phi * u)$. 
The linearization of $A_{i}$ about a function $w \in\left(C^{2+\alpha}(\mathbb{R})\right)^{n}$ is given by the expression

$$
\begin{gathered}
L_{i} u=A_{i}^{\prime}(w) u=\left.\frac{d}{d t}\left[A_{i}(w+t u)\right]\right|_{t=0}=\frac{d}{d t}\left[\alpha_{i}\left(w_{i}^{\prime \prime}+t u_{i}^{\prime \prime}\right)+c\left(w_{i}^{\prime}+t u_{i}^{\prime}\right)+\right. \\
\left.+F_{i}\left(w_{1}+t u_{1}, \ldots, w_{n}+t u_{n}, \varphi_{i 1} *\left(w_{1}+t u_{1}\right), \ldots, \varphi_{i n} *\left(w_{n}+t u_{n}\right)\right)\right]\left.\right|_{t=0} .
\end{gathered}
$$

Hence the operator $L_{i}$ can be written as

$$
\begin{aligned}
L_{i} u= & \alpha_{i} u_{i}^{\prime \prime}+c u_{i}^{\prime}+\sum_{j=1}^{n} \frac{\partial F_{i}}{\partial u_{j}}\left(w_{1}, \ldots, w_{n}, \varphi_{i 1} * w_{1}, \ldots, \varphi_{i n} * w_{n}\right) u_{j}+ \\
& +\sum_{j=1}^{n} \frac{\partial F_{i}}{\partial U_{i j}}\left(w_{1}, \ldots, w_{n}, \varphi_{i 1} * w_{1}, \ldots, \varphi_{i n} * w_{n}\right)\left(\varphi_{i j} * u_{j}\right),
\end{aligned}
$$

where $U_{i j}, j=1, \ldots, n$ denote the variables $n+1, \ldots, 2 n$ of $F_{i}$.

Let $L u=\left(L_{1} u, \ldots, L_{n} u\right), L:\left(C^{2+\alpha}(\mathbb{R})\right)^{n} \rightarrow\left(C^{\alpha}(\mathbb{R})\right)^{n}$. Denote by

$$
\frac{\partial F}{\partial u}=\left(\begin{array}{ccc}
\frac{\partial F_{1}}{\partial u_{1}} & \cdots & \frac{\partial F_{1}}{\partial u_{n}} \\
\cdots & \cdots & \cdots \\
\frac{\partial F_{n}}{\partial u_{1}} & \cdots & \frac{\partial F_{n}}{\partial u_{n}}
\end{array}\right)
$$

the matrix of the derivatives of $F_{1}, \ldots, F_{n}$ with respect to the variables $u_{1}, \ldots, u_{n}$ and by $G$ the operator $G u=\left(G_{1} u, \ldots, G_{n} u\right)^{T}$ with

$$
G_{i} u=\sum_{j=1}^{n} \frac{\partial F_{i}}{\partial U_{i j}}\left(w_{1}, \ldots, w_{n}, \varphi_{i 1} * w_{1}, \ldots, \varphi_{i n} * w_{n}\right)\left(\varphi_{i j} * u_{j}\right),
$$

for $i=1, \ldots, n$. Then $L$ can be written under the form

$$
L u=B u^{\prime \prime}+c u^{\prime}+\frac{\partial F}{\partial u}(w, \Phi * w) u+G u .
$$

We study the normal solvability of the operator $L$ with the aid of its limiting operators. To this end, consider the shifted functions $w_{j}\left(x+x_{m}\right), j=1, \ldots, n$, where $x_{m} \in \mathbb{R}$ is an arbitrary sequence such that $\left|x_{m}\right| \rightarrow \infty$. Since $w_{j}\left(x+x_{m}\right)$ is a bounded sequence in $C^{2+\alpha}(\mathbb{R})$, we can choose a convergent subsequence, for which we keep the same notation, $w_{j}\left(x+x_{m}\right) \rightarrow \widehat{w}_{j}(x)$ as $m \rightarrow \infty$, uniformly on every bounded interval. In addition, we have $\left(\varphi_{i j} * w_{j}\right)\left(x+x_{m}\right) \rightarrow$ $\left(\varphi_{i j} * \widehat{w}_{j}\right)(x)$ as $m \rightarrow \infty$, uniformly on bounded intervals. Indeed, by the change of variable $x_{m}-y=-z$, we get

$$
\begin{gathered}
\left(\varphi_{i j} * w_{j}\right)\left(x+x_{m}\right)=\int_{-\infty}^{\infty} \varphi_{i j}\left(x+x_{m}-y\right) w_{j}(y) d y= \\
=\int_{-\infty}^{\infty} \varphi_{i j}(x-z) w_{j}\left(z+x_{m}\right) d z \rightarrow \int_{-\infty}^{\infty} \varphi_{i j}(x-z) \widehat{w}_{j}(z) d z=\left(\varphi_{i j} * \widehat{w}_{j}\right)(x),
\end{gathered}
$$


uniformly on bounded intervals. Since $F \in C^{1}\left(\mathbb{R}^{2 n}\right)$, we infer that

$$
\begin{aligned}
\frac{\partial F_{i}}{\partial u_{j}}\left(w_{1}(x\right. & \left.\left.+x_{m}\right), \ldots, w_{n}\left(x+x_{m}\right),\left(\varphi_{i 1} * w_{1}\right)\left(x+x_{m}\right), \ldots,\left(\varphi_{i n} * w_{n}\right)\left(x+x_{m}\right)\right) \rightarrow \\
& \rightarrow \frac{\partial F_{i}}{\partial u_{j}}\left(\widehat{w}_{1}(x), \ldots, \widehat{w}_{n}(x),\left(\varphi_{i 1} * \widehat{w}_{1}\right)(x), \ldots,\left(\varphi_{i n} * \widehat{w}_{n}\right)(x)\right)
\end{aligned}
$$

and

$$
\begin{aligned}
\frac{\partial F_{i}}{\partial U_{i j}}\left(w_{1}(x\right. & \left.\left.+x_{m}\right), \ldots, w_{n}\left(x+x_{m}\right),\left(\varphi_{i 1} * w_{1}\right)\left(x+x_{m}\right), \ldots,\left(\varphi_{i n} * w_{n}\right)\left(x+x_{m}\right)\right) \rightarrow \\
\rightarrow & \frac{\partial F_{i}}{\partial U_{i j}}\left(\widehat{w}_{1}(x), \ldots, \widehat{w}_{n}(x),\left(\varphi_{i 1} * \widehat{w}_{1}\right)(x), \ldots,\left(\varphi_{i n} * \widehat{w}_{n}\right)(x)\right),
\end{aligned}
$$

for $i, j \in\{1, \ldots, n\}$, uniformly on bounded intervals.

Now we define the limiting operators $\widehat{L}_{i}$ associated to each $L_{i}$ by replacing the coefficients of $L_{i}$ with their limiting values:

$$
\begin{gathered}
\widehat{L}_{i} u=\alpha_{i} u_{i}^{\prime \prime}+c u_{i}^{\prime}+\sum_{j=1}^{n} \frac{\partial F_{i}}{\partial u_{j}}\left(\widehat{w}_{1}, \ldots, \widehat{w}_{n}, \varphi_{i 1} * \widehat{w}_{1}, \ldots, \varphi_{i n} * \widehat{w}_{n}\right) u_{j}+ \\
+\sum_{j=1}^{n} \frac{\partial F_{i}}{\partial U_{i j}}\left(\widehat{w}_{1}, \ldots, \widehat{w}_{n}, \varphi_{i 1} * \widehat{w}_{1}, \ldots, \varphi_{i n} * \widehat{w}_{n}\right) \int_{-\infty}^{\infty} \varphi_{i j}(x-y) u_{j}(y) d y, i=1, \ldots, n .
\end{gathered}
$$

The limiting operator $\widehat{L}$ associated to $L$ is defined through $\widehat{L} u=\left(\widehat{L}_{1} u, \ldots, \widehat{L}_{n} u\right)$. Because of the different possibilities of choosing the sequence $x_{m}$ (such that $\left|x_{m}\right| \rightarrow \infty$ ) and the convergent subsequence of $w_{j}\left(x+x_{m}\right)$, we obtain generally a family of limiting operators $\widehat{L}_{i}, i=1, \ldots, n$ and $\widehat{L}$.

\subsection{Normal solvability}

We study the normal solvability of the operator $L$ introduced in (2.7). We will also prove that the dimension of its kernel is finite. We state first an estimate for the solution of the equation $L u=f$, which is an extension of Lemma 2.1 from [1] to the case of systems.

Lemma 2.1. Assume that $\varphi_{i j} \in L^{1}(\mathbb{R}), i, j=1, \ldots, n$. If $u$ is an arbitrary solution of the equation $L u=f$, where $f \in\left(C^{\alpha}(\mathbb{R})\right)^{n}$, then there exists a constant $C>0$ independent of $u$ such that

$$
\|u\|_{\left(C^{2+\alpha}(\mathbb{R})\right)^{n}} \leq C\left(\|L u\|_{\left(C^{\alpha}(\mathbb{R})\right)^{n}}+\|u\|_{(C(\mathbb{R}))^{n}}\right) .
$$


Proof. Denote by $E:\left(C^{2+\alpha}(\mathbb{R})\right)^{n} \rightarrow\left(C^{\alpha}(\mathbb{R})\right)^{n}$ the elliptic operator defined through $E u=$ $B u^{\prime \prime}+c u^{\prime}+\frac{\partial F}{\partial u}(w, \Phi * w) u$ and by $E_{i}, i=1, \ldots, n$ its component operators, i. e.

$$
E_{i} u=\alpha_{i} u_{i}^{\prime \prime}+c u_{i}^{\prime}+\sum_{j=1}^{n} \frac{\partial F_{i}}{\partial u_{j}}\left(w_{1}, \ldots, w_{n}, \varphi_{i 1} * w_{1}, \ldots, \varphi_{i n} * w_{n}\right) u_{j} .
$$

It is known that for such operators the Schauder estimate holds (see, e.g., [10]):

$$
\|u\|_{\left(C^{2+\alpha}(\mathbb{R})\right)^{n}} \leq \widetilde{C}\left(\|E u\|_{\left(C^{\alpha}(\mathbb{R})\right)^{n}}+\|u\|_{(C(\mathbb{R}))^{n}}\right),
$$

where $\widetilde{C}>0$ is a constant independent of $u \in\left(C^{2+\alpha}(\mathbb{R})\right)^{n}$. From $(2.5)$ we see that $L_{i}(i=$ $1, \ldots, n)$ can be written under the form $L_{i} u=E_{i} u+G_{i} u$, with $G_{i}$ defined through (2.6). Then $L u=E u+G u$.

Let $u$ be a solution of the equation $L u=f$, for $f=\left(f_{1}, \ldots, f_{n}\right)$. Then $E u=f-G u$, that is

$$
E_{i} u=f_{i}-\sum_{j=1}^{n} \frac{\partial F_{i}}{\partial U_{i j}}\left(w_{1}, \ldots, w_{n}, \varphi_{i 1} * w_{1}, \ldots, \varphi_{i n} * w_{n}\right) g_{i j}
$$

where $g_{i j}(x)=\left(\varphi_{i j} * u_{j}\right)(x)=\int_{-\infty}^{\infty} \varphi_{i j}(x-y) u_{j}(y) d y$.

The boundedness of $w$ on $\mathbb{R}$, together with the hypothesis $\varphi_{i j} \in L^{1}(\mathbb{R}), i, j=1, \ldots, n$, leads to the estimate

$$
\left|\left(\varphi_{i j} * w_{j}\right)(x)\right| \leq\left\|\varphi_{i j}\right\|_{L^{1}(\mathbb{R})}|| w_{j} \|_{C(\mathbb{R})} \leq m,(\forall) x \in \mathbb{R},
$$

where $m>0$ is a constant. Since $F \in C^{1}\left(\mathbb{R}^{2 n}\right)$, it follows from $(2.12)$ that

$$
\|u\|_{\left(C^{2+\alpha}(\mathbb{R})\right)^{n}} \leq \widetilde{C}\left(\|f\|_{\left(C^{\alpha}(\mathbb{R})\right)^{n}}+M \max _{i, j=1, \ldots, n}\left\|g_{i j}\right\|_{\left(C^{\alpha}(\mathbb{R})\right)^{n}}+\|u\|_{(C(\mathbb{R}))^{n}}\right),
$$

with $M>0$ independent of $u$. For $g_{i j}$ we have the estimate

$$
\frac{\left|g_{i j}\left(x_{1}\right)-g_{i j}\left(x_{2}\right)\right|}{\left|x_{1}-x_{2}\right|^{\alpha}} \leq \int_{-\infty}^{\infty}\left|\varphi_{i j}(y)\right| \frac{\left|u_{j}\left(x_{1}-y\right)-u_{j}\left(x_{2}-y\right)\right|}{\left|x_{1}-x_{2}\right|^{\alpha}} d y \leq\left.|| \varphi_{i j}\right|_{L^{1}(\mathbb{R})}|| u_{j} \|_{C^{\alpha}(\mathbb{R})} .
$$

But it is known that for every $\varepsilon>0$, there exists $C_{\varepsilon}>0$ such that

$$
\|u\|_{C^{\alpha}(\mathbb{R})} \leq \varepsilon\|u\|_{C^{2+\alpha}(\mathbb{R})}+C_{\varepsilon}\|u\|_{C(\mathbb{R})},(\forall) u \in C^{2+\alpha}(\mathbb{R})
$$

(see, e.g., [10]), so $\left\|g_{i j}\right\|_{\left(C^{\alpha}(\mathbb{R})\right)^{n}} \leq \widetilde{M}\left(\varepsilon\left\|u_{j}\right\|_{C^{2+\alpha}(\mathbb{R})}+C_{\varepsilon}\left\|u_{j}\right\|_{C(\mathbb{R})}\right)$. We fix $\varepsilon>0$ sufficiently small such that $\widetilde{C} M \widetilde{M} \varepsilon \max _{i, j=1, \ldots, n}\left\|\varphi_{i j}\right\|_{L^{1}(\mathbb{R})}<1$. The claim follows by introducing these estimates into (2.13).

Now we are ready to state the main result of this section. We work under the following hypothesis.

Condition NS. The limiting equations $\widehat{L} u=0$ do not have nonzero solutions in $\left(C^{2+\alpha}(\mathbb{R})\right)^{n}$. 
Theorem 2.2. If Condition NS holds, then $L$ is a normally solvable operator and its kernel ker $L$ has a finite dimension.

Proof. Consider a sequence $f^{k}=\left(f_{1}^{k}, \ldots, f_{n}^{k}\right) \in \operatorname{Im} L$ such that $f^{k} \rightarrow f$ in $\left(C^{\alpha}(\mathbb{R})\right)^{n}, f=$ $\left(f_{1}, \ldots, f_{n}\right)$ and $u^{k}=\left(u_{1}^{k}, \ldots, u_{n}^{k}\right) \in\left(C^{2+\alpha}(\mathbb{R})\right)^{n}$ such that $L u^{k}=f^{k}$.

Part I. Suppose that $u^{k}$ is bounded in $\left(C^{2+\alpha}(\mathbb{R})\right)^{n}$, say $\left\|u^{k}\right\|_{\left(C^{2+\alpha}(\mathbb{R})\right)^{n}} \leq M$. Then $u^{k}$ admits at least a subsequence $u^{k_{m}}$ which is convergent to a limiting function $u^{0}=\left(u_{1}^{0}, \ldots, u_{n}^{0}\right)$, uniformly on every bounded interval of $x$ :

$$
\sup _{x \in I}\left\|u^{k_{m}}(x)-u^{0}(x)\right\| \rightarrow 0, \text { as } m \rightarrow \infty,
$$

for any fixed bounded interval $I$. Here $\|\cdot\|$ is the Euclidian norm in $\mathbb{R}^{n}$.

By a diagonalization process, we prolong $u^{0}$ to all $\mathbb{R}$. It is clear that $\sup _{x \in \mathbb{R}}\left\|u^{0}(x)\right\| \leq M$. Passing to the limit as $m \rightarrow \infty$ in the system $L u^{k_{m}}=f^{k_{m}}$, with $L=\left(L_{1}, \ldots, L_{n}\right)$ given by (2.7), we arrive at $L u^{0}=f$.

We prove now that $u^{k_{m}} \rightarrow u^{0}$ as $m \rightarrow \infty$, uniformly with respect to $x$ on the whole real axis. To do this, we set $v^{m}=u^{k_{m}}-u^{0}$ and note that $v^{m} \rightarrow 0$ as $m \rightarrow \infty$ uniformly on each bounded interval of $x$. Suppose that $v^{m}$ does not tend to 0 uniformly on $\mathbb{R}$. Hence there exists an unbounded sequence $x_{m} \in \mathbb{R}, x_{m} \rightarrow \infty$ such that $\left\|v^{m}\left(x_{m}\right)\right\| \geq \varepsilon>0$. Equalities $L u^{k_{m}}=f^{k_{m}}$ and $L u^{0}=f$ imply that

$$
L\left(u^{k_{m}}-u^{0}\right)=f^{k_{m}}-f,
$$

or, in view of (2.7),

$$
B\left(v^{m}\right)^{\prime \prime}+c\left(v^{m}\right)^{\prime}+\frac{\partial F}{\partial u}(w, \Phi * w) v^{m}+G v^{m}=f^{k_{m}}-f
$$

with $G$ given by $(2.6)$.

Denote $V^{m}(x)=v^{m}\left(x+x_{m}\right)=u^{k_{m}}\left(x+x_{m}\right)-u^{0}\left(x+x_{m}\right)$. Then $V^{m}$ verifies the equation

$$
\begin{gathered}
B\left(V^{m}\right)^{\prime \prime}(x)+c\left(V^{m}\right)^{\prime}(x)+\frac{\partial F}{\partial u}(w, \Phi * w)\left(x+x_{m}\right) V^{m}(x)+ \\
+G v^{m}\left(x+x_{m}\right)=f^{k_{m}}\left(x+x_{m}\right)-f\left(x+x_{m}\right)
\end{gathered}
$$

or in detail, for every $i=1, \ldots, n$,

$$
\begin{gathered}
\alpha_{i}\left(V_{i}^{m}\right)^{\prime \prime}(x)+c\left(V_{i}^{m}\right)^{\prime}(x)+\sum_{j=1}^{n} \frac{\partial F_{i}}{\partial u_{j}}\left(w_{1}, \ldots, w_{n}, \varphi_{i 1} * w_{1}, \ldots, \varphi_{i n} * w_{n}\right)\left(x+x_{m}\right) V_{j}^{m}(x)+ \\
+\sum_{j=1}^{n} \frac{\partial F_{i}}{\partial U_{i j}}\left(w_{1}, \ldots, w_{n}, \varphi_{i 1} * w_{1}, \ldots, \varphi_{i n} * w_{n}\right)\left(x+x_{m}\right)\left(\varphi_{i j} * v_{j}^{m}\right)\left(x+x_{m}\right)= \\
=f_{i}^{k_{m}}\left(x+x_{m}\right)-f_{i}\left(x+x_{m}\right) .
\end{gathered}
$$


Using the change of variable $x_{m}-y=-z$, we find

$$
\left(\varphi_{i j} * v_{j}^{m}\right)\left(x+x_{m}\right)=\int_{-\infty}^{\infty} \varphi_{i j}\left(x+x_{m}-y\right) v_{j}^{m}(y) d y=\int_{-\infty}^{\infty} \varphi_{i j}(x-z) v_{j}^{m}\left(z+x_{m}\right) d z .
$$

Then the above equation becomes

$$
\begin{gathered}
\alpha_{i}\left(V_{i}^{m}\right)^{\prime \prime}(x)+c\left(V_{i}^{m}\right)^{\prime}(x)+\sum_{j=1}^{n} \frac{\partial F_{i}}{\partial u_{j}}\left(w_{1}, \ldots, w_{n}, \varphi_{i 1} * w_{1}, \ldots, \varphi_{i n} * w_{n}\right)\left(x+x_{m}\right) V_{j}^{m}(x)+ \\
+\sum_{j=1}^{n} \frac{\partial F_{i}}{\partial U_{i j}}\left(w_{1}, \ldots, w_{n}, \varphi_{i 1} * w_{1}, \ldots, \varphi_{i n} * w_{n}\right)\left(x+x_{m}\right) \int_{-\infty}^{\infty} \varphi_{i j}(x-y) V_{j}^{m}(y) d y= \\
=f_{i}^{k_{m}}\left(x+x_{m}\right)-f_{i}\left(x+x_{m}\right) .
\end{gathered}
$$

Function $V^{m}$ also satisfies

$$
\left\|V^{m}(0)\right\|=\left\|v^{m}\left(x_{m}\right)\right\| \geq \varepsilon>0 .
$$

Since $\left\|V^{m}\right\|_{\left(C^{2+\alpha}(\mathbb{R})\right)^{n}} \leq M$, it follows that $V^{m}$ is convergent at least on a subsequence $\left\{m_{l}\right\}_{l=1}^{l=\infty}$ to a function $V^{0}$, uniformly on bounded intervals $I$ of $x$. The limit function $V^{0}$ can be prolonged on $\mathbb{R}$ such that $V^{0} \in\left(C^{2+\alpha}(\mathbb{R})\right)^{n}$. Let $V^{0}=\left(V_{1}^{0}, \ldots, V_{n}^{0}\right)$.

Now we may pass to the limit in $(2.15)$ and $(2.16)$ on the subsequence $m_{l}$ uniformly on bounded intervals $I$ of $x$. One arrives at

$$
\begin{gathered}
\alpha_{i}\left(V_{i}^{0}\right)^{\prime \prime}(x)+c\left(V_{i}^{0}\right)^{\prime}(x)+\sum_{j=1}^{n} \frac{\partial F_{i}}{\partial u_{j}}\left(\widehat{w}_{1}, \ldots, \widehat{w}_{n}, \varphi_{i 1} * \widehat{w}_{1}, \ldots, \varphi_{i n} * \widehat{w}_{n}\right)(x) V_{j}^{0}(x)+ \\
+\sum_{j=1}^{n} \frac{\partial F_{i}}{\partial U_{i j}}\left(\widehat{w}_{1}, \ldots, \widehat{w}_{n}, \varphi_{i 1} * \widehat{w}_{1}, \ldots, \varphi_{i n} * \widehat{w}_{n}\right)(x) \int_{-\infty}^{\infty} \varphi_{i j}(x-y) V_{j}^{0}(y) d y=0
\end{gathered}
$$

and $\left\|V^{0}(0)\right\| \geq \varepsilon>0$. Thus $V^{0}=\left(V_{1}^{0}, \ldots, V_{n}^{0}\right)$ is a nonzero solution of the limiting operator $\widehat{L}$ from $(2.10)$, i. e. Condition NS is not satisfied. This contradiction proves that $u^{k_{m}} \rightarrow u^{0}$ as $m \rightarrow \infty$ in $(C(\mathbb{R}))^{n}$. Moreover, $u^{k_{m}} \rightarrow u^{0}$ as $m \rightarrow \infty$ in $\left(C^{2+\alpha}(\mathbb{R})\right)^{n}$. Indeed, applying Lemma 1 to equation $(2.14)$, we deduce that

$$
\left\|u^{k_{m}}-u^{0}\right\|_{\left(C^{2+\alpha}(\mathbb{R})\right)^{n}} \leq C\left(\left\|f^{k_{m}}-f\right\|_{\left(C^{\alpha}(\mathbb{R})\right)^{n}}+\left\|u^{k_{m}}-u^{0}\right\|_{(C(\mathbb{R}))^{n}}\right) .
$$

Since $f^{k_{m}} \rightarrow f$ in $\left(C^{\alpha}(\mathbb{R})\right)^{n}$ and $u^{k_{m}} \rightarrow u^{0}$ in $(C(\mathbb{R}))^{n}$, we conclude that $u^{k_{m}} \rightarrow u^{0}$ in $\left(C^{2+\alpha}(\mathbb{R})\right)^{n}$. Therefore, there is a convergent subsequence $u^{k_{m}} \rightarrow u^{0}$ in $\left(C^{2+\alpha}(\mathbb{R})\right)^{n}$ such that $L u^{0}=f$, i. e. $f \in \operatorname{Im} L$.

Part II. For $f^{k}=0$ and $u^{k} \in\left(C^{2+\alpha}(\mathbb{R})\right)^{n}, u^{k}$ bounded in $\left(C^{2+\alpha}(\mathbb{R})\right)^{n}$, such that $L u^{k}=0$, the previous reasoning leads to the existence of a convergent sequence $u^{k_{m}} \rightarrow u^{0}$ in $\left(C^{2+\alpha}(\mathbb{R})\right)^{n}$ such that $L u^{0}=0$. This implies that ker $L$ is locally compact and consequently it has a finite dimension. 
Part III. Let $u^{k}$ be unbounded in $\left(C^{2+\alpha}(\mathbb{R})\right)^{n}$ such that $L u^{k}=f^{k}$. Since ker $L$ is finite dimensional, its supplement $(\operatorname{ker} L)^{\perp}$ is closed. Then $u^{k}$ can be written as $u^{k}=v^{k}+z^{k}$ with $v^{k} \in \operatorname{ker} L$ and $z^{k} \in(\operatorname{ker} L)^{\perp}$. It follows that $L z^{k}=f^{k}$. We show that $z^{k}$ is bounded in $\left(C^{2+\alpha}(\mathbb{R})\right)^{n}$.

Indeed, if it is not the case, we take $\widetilde{z}^{k}=z^{k} /\left\|z^{k}\right\|$ and $\widetilde{f}^{k}=f^{k} /\left\|z^{k}\right\|$. Then $L \widetilde{z}^{k}=\widetilde{f}^{k}$ and $\widetilde{f}^{k} \rightarrow 0$. By Part I, we can choose a convergent subsequence $\widetilde{z}^{k_{m}} \rightarrow \widetilde{z}^{0}$. Letting $m \rightarrow \infty$ in the equation $L \widetilde{z}^{k_{m}}=\widetilde{f}^{k_{m}}$, one derives that $\widetilde{z}^{0} \in \operatorname{ker} L$. This contradiction shows that in fact, $z^{k}$ is bounded in $\left(C^{2+\alpha}(\mathbb{R})\right)^{n}$. Applying Part I for the equation $L z^{k}=f^{k}, z^{k}$ bounded, it follows that $f \in \operatorname{Im} L$.

Therefore we have proved that $\operatorname{Im} L$ is closed and $\operatorname{ker} L$ is finite dimensional. The theorem is proved.

\subsection{Fredholm property}

We go on with the study of the Fredholm property for the operator $L$ and with the computation of the index. To this end, some auxiliary results are needed. We briefly recall the definition of some norms in function spaces which were introduced in [12].

Consider a partition of unity on $\mathbb{R},\left\{\psi_{k}\right\}$, with $\psi_{k} \in C^{\infty}(\mathbb{R}), \psi_{k}(x)=1$ for $x \in(k, k+1)$ and $\psi_{k}(x)=0$ for $x \in(-\infty, k-1)$ and $x \in(k+2, \infty)$. For $\rho>0$ and an integer $s \geq 0$, we can define the norm $W_{\infty, \rho}^{s, 2}(\mathbb{R})$ of the function $v: \mathbb{R} \rightarrow \mathbb{R}$ by

$$
\||| v \mid\|_{W_{\infty, \rho}^{s, 2}(\mathbb{R})}=\sup _{k \in \mathbb{Z}}\left(\left\|\psi_{k} v\right\|_{W^{s, 2}(\mathbb{R})}+|\rho|^{s}\left\|\psi_{k} v\right\|_{L^{2}(\mathbb{R})}\right)
$$

and the norm $\left(W_{\infty, \rho}^{s, 2}(\mathbb{R})\right)^{n}$ of the function $u=\left(u_{1}, \ldots, u_{n}\right): \mathbb{R} \rightarrow \mathbb{R}^{n}$ by

$$
\left\||| u\left|\|_{\left(W_{\infty, \rho}^{s, 2}(\mathbb{R})\right)^{n}}=\sum_{j=1}^{n}\right||| u_{j}||_{W_{\infty, \rho}^{s, 2}(\mathbb{R})} .\right.
$$

The following result was proved in [12].

Lemma 2.3. Let $L(\rho): W_{\infty, \rho}^{s, 2}(\mathbb{R}) \rightarrow L_{\infty, \rho}^{2}(\mathbb{R})$ be uniformly elliptic operator with a parameter. Then there exists $\rho_{0}>0$ such that for every $\rho \geq \rho_{0}$ and every $f \in L_{\infty, \rho}^{2}(\mathbb{R})$, the solution $v$ of the equation $L(\rho) v=f$ satisfies the estimate

$$
\left\||| v||_{W_{\infty, \rho}^{s, 2}(\mathbb{R})} \leq K \mid\right\| f \|_{L_{\infty, \rho}^{2}(\mathbb{R})}
$$

for some constant $K>0$.

We note that the norm $\||\cdot|\|_{L_{\infty, \rho}^{2}(\mathbb{R})}$ is given by $(2.17)$ for $s=0$.

Lemma 2.4. Let $L_{1}, L_{2}, L_{\tau}:\left(C^{2+\alpha}(\mathbb{R})\right)^{n} \rightarrow\left(C^{\alpha}(\mathbb{R})\right)^{n}, \tau \in[0,1]$ be the operators defined through $L_{1} u=L u-\rho u, L_{2} u=B u^{\prime \prime}-\rho u, L_{\tau} u=(1-\tau) L_{1} u+\tau L_{2} u$, where $\rho \geq 0$ is a parameter. Denote by $\widehat{L}_{\tau}$ the limiting operators associated to $L_{\tau}$. Suppose that all functions $\varphi_{i j}$ satisfy the condition 


$$
\sum_{k=-\infty}^{\infty}\left(\int_{k}^{k+1} \varphi_{i j}^{2}(s) d s\right)^{1 / 2}<+\infty
$$

Then there is a sufficiently large $\rho \geq 0$ such that the limiting equations $\widehat{L}_{\tau} u=0$ do not have nonzero solutions for any $\tau \in[0,1]$.

Proof. The operator $L_{\tau}$ can be written explicitly in the form

$$
L_{\tau} u=B u^{\prime \prime}+(1-\tau) c u^{\prime}+\left[(1-\tau) \frac{\partial F}{\partial u}(w, \Phi * w)-\rho\right] u+(1-\tau) G u .
$$

Let $\widehat{L}_{\tau}$ be one of its limiting operators. The i-th component of $\widehat{L}_{\tau}$ is

$$
\begin{aligned}
& \left(\widehat{L}_{\tau} u\right)_{i}=\alpha_{i} u_{i}^{\prime \prime}+(1-\tau) c u_{i}^{\prime}+(1-\tau) \sum_{j=1}^{n} \frac{\partial F_{i}}{\partial u_{j}}\left(\widehat{w}_{1}, \ldots, \widehat{w}_{n}, \varphi_{i 1} * \widehat{w}_{1}, \ldots, \varphi_{i n} * \widehat{w}_{n}\right) u_{j}-\rho u_{i}+ \\
& +(1-\tau) \sum_{j=1}^{n} \frac{\partial F_{i}}{\partial U_{i j}}\left(\widehat{w}_{1}, \ldots, \widehat{w}_{n}, \varphi_{i 1} * \widehat{w}_{1}, \ldots, \varphi_{i n} * \widehat{w}_{n}\right) \int_{-\infty}^{\infty} \varphi_{i j}(x-y) u_{j}(y) d y, i=1, \ldots, n .
\end{aligned}
$$

We show the existence of some $\rho>0$ sufficiently large such that the equation $\widehat{L}_{\tau} u=0$ does not have nonzero solutions for any $\tau \in[0,1]$.

Let $L_{3}:\left(C^{2+\alpha}(\mathbb{R})\right)^{n} \rightarrow\left(C^{\alpha}(\mathbb{R})\right)^{n}$ be the elliptic operator defined by

$$
L_{3} u=B u^{\prime \prime}+(1-\tau) c u^{\prime}+(1-\tau) \frac{\partial F}{\partial u}(\widehat{w}, \Phi * \widehat{w}) u
$$

and $f=\left(f_{1}, \ldots, f_{n}\right)$, where

$$
f_{i}=\sum_{j=1}^{n} \frac{\partial F_{i}}{\partial U_{i j}}\left(\widehat{w}_{1}, \ldots, \widehat{w}_{n}, \varphi_{i 1} * \widehat{w}_{1}, \ldots, \varphi_{i n} * \widehat{w}_{n}\right) \int_{-\infty}^{\infty} \varphi_{i j}(x-y) u_{j}(y) d y .
$$

If $L_{3} u$ has the components $\left(L_{3} u\right)_{i}, i=1, \ldots, n$, then

$$
\left(L_{3} u\right)_{i}=\alpha_{i} u_{i}^{\prime \prime}+(1-\tau) c u_{i}^{\prime}+(1-\tau) \sum_{j=1}^{n} \frac{\partial F_{i}}{\partial u_{j}}\left(\widehat{w}_{1}, \ldots, \widehat{w}_{n}, \varphi_{i 1} * \widehat{w}_{1}, \ldots, \varphi_{i n} * \widehat{w}_{n}\right) u_{j}
$$

The limiting equations $\widehat{L}_{\tau} u=0$ can be written as

$$
L_{3} u-\rho u=(\tau-1) f .
$$

The $i$-the equation of this system has the form

$$
\alpha_{i} u_{i}^{\prime \prime}+(1-\tau) c u_{i}^{\prime}+(1-\tau) \frac{\partial F_{i}}{\partial u_{i}}\left(\widehat{w}_{1}, \ldots, \widehat{w}_{n}, \varphi_{i 1} * \widehat{w}_{1}, \ldots, \varphi_{i n} * \widehat{w}_{n}\right) u_{i}-\rho u_{i}=
$$




$$
=(\tau-1)\left[f_{i}+\sum_{\substack{j=1 \\ j \neq i}}^{n} \frac{\partial F_{i}}{\partial u_{j}}\left(\widehat{w}_{1}, \ldots, \widehat{w}_{n}, \varphi_{i 1} * \widehat{w}_{1}, \ldots, \varphi_{i n} * \widehat{w}_{n}\right) u_{j}\right] .
$$

The operator in the left-hand side of this equation is uniformly elliptic with a parameter $\rho$, so we may apply Lemma 2.3 for $s=2$. Therefore there are constants $K_{1}>0$ and $\rho_{0}>0$ such that $(\forall) \rho \geq \rho_{0}$ and $(\forall) \tau \in[0,1]$,

$$
\left.\left\|\left.\left|u_{i}\right|\right|_{W_{\infty, \rho}^{2,2}(\mathbb{R})} \leq\left.||\left|f_{i}\right|\right|_{L_{\infty, \rho}^{2}(\mathbb{R})}+K_{1} \sum_{j=1}^{n}\right\|\left|u_{j}\right|\right|_{L_{\infty, \rho}^{2}(\mathbb{R})},
$$

for any solution $u_{i}$ of the equation (2.21). We have used the boundedness of

$$
\frac{\partial F_{i}}{\partial U_{j}}\left(\widehat{w}_{1}, \ldots, \widehat{w}_{n}, \varphi_{i 1} * \widehat{w}_{1}, \ldots, \varphi_{i n} * \widehat{w}_{n}\right),
$$

which follows from the assumption that $F \in C^{1}(\mathbb{R})$ and the boundedness of $\widehat{w}$. In view of $(2.17)$ this implies that

$$
\rho^{2} \sup _{k \in \mathbb{Z}}\left\|\psi_{k} u_{i}\right\|_{L^{2}(\mathbb{R})} \leq \sup _{k \in \mathbb{Z}}\left\|\psi_{k} f_{i}\right\|_{L^{2}(\mathbb{R})}+K_{1} \sup _{k \in \mathbb{Z}} \sum_{j=1}^{n}\left\|\psi_{k} u_{j}\right\|_{L^{2}(\mathbb{R})} .
$$

We now prove that each component $f_{i}$ of $f$ satisfies the inequality

$$
\sup _{k \in \mathbb{Z}}\left\|\psi_{k} f_{i}\right\|_{L^{2}(\mathbb{R})} \leq K_{2} \sup _{k \in \mathbb{Z}} \sum_{j=1}^{n}\left\|\psi_{k} u_{j}\right\|_{L^{2}(\mathbb{R})}
$$

where $K_{2}>0$ is a constant. To do this, we set

$$
g_{i j}(x)=\int_{-\infty}^{\infty} \varphi_{i j}(x-y) u_{j}(y) d y .
$$

Then for a given $k \in \mathbb{Z}$, we have

$$
\begin{aligned}
& \int_{-\infty}^{\infty} \psi_{k}^{2}(x) g_{i j}^{2}(x) d x=\int_{k}^{k+1} \psi_{k}^{2}(x)\left(\int_{-\infty}^{\infty} \varphi_{i j}(x-y) u_{j}(y) d y\right)^{2} d x \\
& \leq \int_{k}^{k+1} \psi_{k}^{2}(x)\left(\sum_{l=-\infty}^{\infty} \int_{l}^{l+1} \psi_{l}(y) \varphi_{i j}(x-y) u_{j}(y) d y\right)^{2} d x \\
& \leq \int_{k}^{k+1} \psi_{k}^{2}(x)\left(\sum_{l=-\infty}^{\infty}\left\|\psi_{l} u_{j}\right\|_{L^{2}(\mathbb{R})}\left\|\varphi_{i j}(x-\cdot)\right\|_{L^{2}(l, l+1)}\right)^{2} d x \\
& \leq \sup _{l \in \mathbb{Z}}\left\|\psi_{l} u_{j}\right\|_{L^{2}(\mathbb{R})}^{2} \int_{k}^{k+1} \psi_{k}^{2}(x)\left(\sum_{l=-\infty}^{\infty}\left\|\varphi_{i j}(x-\cdot)\right\|_{L^{2}(l, l+1)}\right)^{2} d x .
\end{aligned}
$$


Making use of $(2.20)$, we can prove that

$$
K^{2}:=\sup _{k \in \mathbb{Z}} \int_{k}^{k+1} \psi_{k}^{2}(x)\left(\sum_{l=-\infty}^{\infty}\left(\int_{l}^{l+1} \varphi_{i j}^{2}(x-y) d y\right)^{1 / 2}\right)^{2} d x<\infty .
$$

Indeed, suppose that condition (2.20) is satisfied and let $x \in \mathbb{R}$ be given. Then there exist $n \in \mathbb{Z}$ and $t \in[0,1)$ such that $x=n+t$. Next, for any $l \in \mathbb{Z}$ we have

$$
\int_{l}^{l+1} \varphi_{i j}^{2}(x-y) d y=\int_{n-l-1}^{n-l} \varphi_{i j}^{2}(t+s) d s .
$$

Thus we get

$$
\begin{aligned}
& \sum_{l=-\infty}^{\infty}\left(\int_{l}^{l+1} \varphi_{i j}^{2}(x-y) d y\right)^{1 / 2}=\sum_{l=-\infty}^{\infty}\left(\int_{n-l-1}^{n-l} \varphi_{i j}^{2}(t+s) d s\right)^{1 / 2}= \\
& =\sum_{h=-\infty}^{\infty}\left(\int_{h}^{h+1} \varphi_{i j}^{2}(t+s) d s\right)^{1 / 2}=\sum_{h=-\infty}^{\infty}\left(\int_{h+t}^{h+t+1} \varphi_{i j}^{2}(z) d z\right)^{1 / 2}
\end{aligned}
$$

with $h=n-l-1$. But

$$
\begin{gathered}
\left(\int_{h+t}^{h+t+1} \varphi_{i j}^{2}(z) d z\right)^{1 / 2} \leq\left(\int_{h}^{h+1} \varphi_{i j}^{2}(z) d z+\int_{h+1}^{h+2} \varphi_{i j}^{2}(z) d z\right)^{1 / 2} \leq \\
\leq\left(\int_{h}^{h+1} \varphi_{i j}^{2}(z) d z\right)^{1 / 2}+\left(\int_{h+1}^{h+2} \varphi_{i j}^{2}(z) d z\right)^{1 / 2} .
\end{gathered}
$$

Therefore,

$$
\sum_{l=-\infty}^{\infty}\left(\int_{l}^{l+1} \varphi_{i j}^{2}(x-y) d y\right)^{1 / 2} \leq 2 \sum_{h=-\infty}^{\infty}\left(\int_{h}^{h+1} \varphi_{i j}^{2}(z) d z\right)^{1 / 2} .
$$

By (2.20) one arrives at

$$
K^{2} \leq 4\left(\sum_{h=-\infty}^{\infty}\left(\int_{h}^{h+1} \varphi_{i j}^{2}(s) d s\right)^{1 / 2}\right)^{2}<+\infty
$$

Thus (2.25) is proved. In view of (2.24) and the definition of $f_{i}$, we obtain

$$
\sup _{k \in \mathbb{Z}}\left\|\psi_{k} f_{i}\right\|_{L^{2}(\mathbb{R})} \leq K_{3} \sup _{k \in \mathbb{Z}}\left\|\psi_{k} g_{i j}\right\|_{L^{2}(\mathbb{R})} \leq K_{4} \sup _{l \in \mathbb{Z}}\left\|\psi_{l} u_{j}\right\|_{L^{2}(\mathbb{R})},
$$

where $K_{3}, K_{4}$ are positive constants. This implies (2.23).

Combining now $(2.22)$ with $(2.23)$, we find that for all $i \in\{1, \ldots, n\}$,

$$
\rho^{2} \sup _{k \in \mathbb{Z}}\left\|\psi_{k} u_{i}\right\|_{L^{2}(\mathbb{R})} \leq K_{5} \sup _{k \in \mathbb{Z}} \sum_{j=1}^{n}\left\|\psi_{k} u_{j}\right\|_{L^{2}(\mathbb{R})}
$$


where $K_{5}$ is a positive constant. Writing similar estimates for all $i$ and summing them up, we deduce that for $\rho>0$ large enough, the solution of the limiting equation $\widehat{L}_{\tau} u=0$ is $u=0$. The proof is complete.

Remark 2.5. Lemma 2.4 shows that the operator $L_{\tau}$ satisfies Condition NS for $\rho$ large enough. Using this result, we can now state the Fredholm property of $L$ provided that the following stronger hypothesis holds.

Condition NS $(\lambda)$. The limiting equations $\widehat{L} u-\lambda u=0$ associated to $L-\lambda I$, do not admit nonzero solutions in $\left(C^{2+\alpha}(\mathbb{R})\right)^{n}$, for any $\lambda \geq 0$.

Theorem 2.6. If $L$ satisfies Condition NS $(\lambda)$, then $L$ is a Fredholm operator with the zero index.

The proof is analogous to that of Theorem 3.2 in the paper [1] and we omit it. One works with the operators $L_{1}, L_{2}, L_{\tau}$ defined above and uses Lemma 2.4, Remark 2.5, and Theorem 2.2 for the operator $L_{\tau}$. We employ the remark that $L_{\tau}$ is a continuous deformation of $L_{1}$ to the invertible operator $L_{2}$ in the class of normally solvable operators with finite dimensional kernels.

\section{Structural stability of wave solutions}

In this section we consider the equation

$$
B u^{\prime \prime}+c u^{\prime}+F\left(u, \Phi_{\varepsilon} * u\right)=0, \quad x \in \mathbb{R},
$$

where $\varepsilon=\left(\varepsilon_{i j}\right) \in \mathcal{M}_{n}(\mathbb{R})$ and $\Phi_{\varepsilon}$ is defined by

$$
\Phi_{\varepsilon}(y)=\left(\frac{1}{\varepsilon_{i j}} \varphi_{i j}\left(\frac{y}{\varepsilon_{i j}}\right)\right)_{i, j=1, . ., n} \forall y \in \mathbb{R} .
$$

In order to be more precise, we introduce the operators $\widehat{\mathcal{F}}_{i, j}$ for $i, j=1, . ., n$ acting from $C^{2+\alpha}(\mathbb{R}) \times$ $\mathbb{R}$ into $C^{\alpha}(\mathbb{R})$ defined by

$$
\widehat{\mathcal{F}}_{i, j}(\varepsilon, u)=\left\{\begin{array}{l}
u \text { if } \varepsilon=0 \\
\frac{1}{\varepsilon} \int_{\mathbb{R}} \varphi_{i j}\left(\frac{x-y}{\varepsilon}\right) u(y) d y \text { if } \varepsilon \neq 0 .
\end{array}\right.
$$

Next, we construct the operator $\mathcal{F}$ acting from $\mathcal{M}_{n}(\mathbb{R}) \times\left(C^{2+\alpha}(\mathbb{R})\right)^{n}$ into $C^{\alpha}\left(\mathbb{R}, \mathcal{M}_{n}(\mathbb{R})\right)$ defined by

$$
\mathcal{F}(\varepsilon, u)=\left(\widehat{\mathcal{F}}_{i, j}\left(\varepsilon_{i j}, u_{i}\right)\right), \quad \varepsilon=\left(\varepsilon_{i j}\right), \quad u=\left(u_{1}, . ., u_{n}\right) .
$$

Therefore we are concerned with the problem

$$
B u^{\prime \prime}+c u^{\prime}+F(u, \mathcal{F}(\varepsilon, u))=0, \quad x \in \mathbb{R},
$$

with $\varepsilon$ close to the zero matrix. This problem is related to the unperturbed system

$$
\begin{aligned}
& B u^{\prime \prime}+c u^{\prime}+f(u)=0, \quad x \in \mathbb{R}, \\
& u( \pm \infty)=u^{ \pm} .
\end{aligned}
$$


Here the function $f$ is defined by

$$
f(u)=F(u, \bar{\Phi} u), \text { with } \bar{\Phi}=\left(\int_{\mathbb{R}} \varphi_{i j}(y) d y\right)_{i, j=1, . ., n} \in \mathcal{M}_{n}(\mathbb{R}) .
$$

We denote by $\left(c_{0}, u_{0}\right) \in \mathbb{R} \times\left(C^{2+\alpha}(\mathbb{R})\right)^{n}$ a solution of this problem. The structural stability of this solution is strongly related to the properties of the linearized operator $A:\left(C^{2+\alpha}(\mathbb{R})\right)^{n} \rightarrow$ $\left(C^{\alpha}(\mathbb{R})\right)^{n}$ about the solution $u_{0}$. Operator $A$ is defined by the equality:

$$
A u=B u^{\prime \prime}+c_{0} u^{\prime}+f^{\prime}\left(u_{0}(x)\right) u .
$$

We assume that the following condition is satisfied:

Operator $A$ satisfies the Fredholm property and has the zero index $\operatorname{codim} R(A)=p, \quad \operatorname{dim} \operatorname{ker} A=q$,

where $R(A)$ is the range of the operator $A$. Under this assumption, operator $A$ has the index $l=q-p$. Moreover due to the Fredholm property (assumption (3.6)), the solvability conditions for operator $A$ are determined by the formally adjoint operator $A^{*}$ acting from $\left(C^{2+\alpha}(\mathbb{R})\right)^{n}$ into $\left(C^{\alpha}(\mathbb{R})\right)^{n}$ and defined by

$$
A^{*} v=B v^{\prime \prime}+c v^{\prime}+f^{\prime}\left(u_{0}(x)\right)^{*} v \quad \forall v \in\left(C^{2+\alpha}(\mathbb{R})\right)^{n} .
$$

This operator has a $p$-dimensional kernel. Denote by $v_{1}^{*}, \ldots, v_{p}^{*}$ its basis. Then for each $h \in$ $\left(C^{\alpha}(\mathbb{R})\right)^{n}$, the equation $A u=h$ has a solution $u \in\left(C^{2+\alpha}(\mathbb{R})\right)^{n}$ if and only if the function $h$ satisfies the conditions

$$
\int_{\mathbb{R}}\left(h(x), v_{i}^{*}(x)\right) d x=0, \quad \forall i=1, . ., p,
$$

where $($,$) denotes the inner product in \mathbb{R}^{n}$. In order to prove structural stability, we need to satisfy the solvability conditions. For that purpose, we introduce a map $M: \mathbb{R}^{p} \rightarrow \mathcal{M}_{n}(\mathbb{R})$ of the class $C^{1}$ and such that $M(0)=c_{0} I_{n}$ where $I_{n}$ is the identity matrix of the order $n$. Then we now consider the problem with parameters

$$
\begin{aligned}
& B u^{\prime \prime}+M(\tau) u^{\prime}+F(u, \mathcal{F}(\varepsilon, u))=0, \quad x \in \mathbb{R}, \\
& u( \pm \infty)=u^{ \pm}
\end{aligned}
$$

where $\varepsilon \in \mathcal{M}_{n}(\mathbb{R})$.

Then we have the following result:

Theorem 3.1. Let system (3.5) have a solution $\left(c_{0}, u_{0}\right) \in \mathbb{R} \times\left(C^{2+\alpha}(\mathbb{R})\right)^{n}$. Assume moreover that assumption (3.6) is satisfied,

$$
\varphi_{i j} \in L_{+}^{1}(\mathbb{R}), \quad \int_{\mathbb{R}} y^{2} \varphi_{i j}(y) d y<\infty, \quad \forall i, j=1, . ., n
$$


and suppose that the linear system with respect to $\tau \in \mathbb{R}^{p}$

$$
\int_{\mathbb{R}}\left(M^{\prime}(0) \tau u_{0}^{\prime}(x), v_{i}^{*}(x)\right) d x=0 \quad i=1, . ., p
$$

has a unique solution $\tau=0$. Then for each $\varepsilon \in \mathcal{M}_{n}(\mathbb{R})$ such that $\|\varepsilon\|<\epsilon_{0}$ and some $\epsilon_{0}>0$ there exist $\tau(\varepsilon) \in \mathbb{R}^{p}$ and $u(\varepsilon) \in\left(C^{2+\alpha}(\mathbb{R})\right)^{n}$ which satisfy (3.8).

The proof of this result relies on the application of the implicit function theorem. For that purpose, we consider the nonlinear operator $\mathcal{L}$ acting from $\mathbb{R}^{p} \times \mathcal{M}_{n}(\mathbb{R}) \times\left(C^{2+\alpha}(\mathbb{R})\right)^{n}$ into $\left(C^{\alpha}(\mathbb{R})\right)^{n}$ and defined by

$$
\mathcal{L}(\tau, \varepsilon, u)=B u^{\prime \prime}+M(\tau) u^{\prime}+F(u, \mathcal{F}(\varepsilon, u)) .
$$

First of all we recall the following regularity result proved in [1]:

Lemma 3.2. Let $\varphi \in L^{1}(\mathbb{R}, \mathbb{R})$ satisfying

$$
\int_{\mathbb{R}} y^{2}|\varphi(y)| d y<\infty .
$$

Then the operator $\mathcal{G}$ acting from $\mathbb{R} \times C^{2+\alpha}(\mathbb{R})$ into $C^{\alpha}(\mathbb{R})$ and defined by

$$
\mathcal{G}(\varepsilon, u)=\left\{\begin{array}{l}
u \text { if } \varepsilon=0 \\
\frac{1}{\varepsilon} \int_{\mathbb{R}} \varphi\left(\frac{x-y}{\varepsilon}\right) u(y) d y \text { if } \varepsilon \neq 0,
\end{array}\right.
$$

is of the class $C^{1}$.

Proof of Theorem 3.1. Due to assumption (3.9) and Lemma 3.2, the operator $\mathcal{F}$ is of the class $C^{1}$ from $\mathcal{M}_{n}(\mathbb{R}) \times\left(C^{2+\alpha}(\mathbb{R})\right)^{n}$ into $C^{\alpha}\left(\mathbb{R}, \mathcal{M}_{n}(\mathbb{R})\right)$. Therefore the operator $\mathcal{L}$ defined in (3.10) is of the class $C^{1}$ from $\mathbb{R}^{p} \times \mathcal{M}_{n}(\mathbb{R}) \times\left(C^{2+\alpha}(\mathbb{R})\right)^{n}$ into $\left(C^{\alpha}(\mathbb{R})\right)^{n}$. Moreover we have

$$
\mathcal{L}_{(\tau, u)}^{\prime}\left(0,0, u_{0}\right)=A u+M^{\prime}(0) \tau u_{0}^{\prime} .
$$

We are now concerned with the solvability of the problem: find $(\tau, u) \in \mathbb{R}^{p} \times\left(C^{2+\alpha}(\mathbb{R})\right)^{n}$ satisfying

$$
A u=h-M^{\prime}(0) \tau u_{0}^{\prime} \text { with } h \in\left(C^{\alpha}(\mathbb{R})\right)^{n} .
$$

The solvability of this equation relies on (3.7). Thus (3.13) has a solution $u \in\left(C^{2+\alpha}(\mathbb{R})\right)^{n}$ if and only if

$$
\int_{\mathbb{R}}\left(h(x), v_{i}^{*}(x)\right) d x=\int_{\mathbb{R}}\left(M^{\prime}(0) \tau u_{0}^{\prime}(x), v_{i}^{*}(x)\right) d x \quad i=1, . ., p .
$$

Due to the assumption of the theorem, this uniquely provides a value $\tau_{1} \in \mathbb{R}^{p}$ and thus also provides $u_{1} \in\left(C^{2+\alpha}(\mathbb{R})\right)^{n}$ such that $\left(\tau_{1}, u_{1}\right)$ is a solution of (3.13). However equation (3.13) is not uniquely solvable. Indeed each $\left(\tau_{1}, u_{1}+v\right)$ with $v \in \operatorname{ker} A$ is also a solution of (3.13). Therefore equation (3.13) becomes uniquely solvable on the space

$$
\mathbb{R}^{p} \times \widehat{E} \text { with } \widehat{E}=\left\{u \in\left(C^{2+\alpha}(\mathbb{R})\right)^{n}, \quad \int_{\mathbb{R}}\left(u(x), v_{j}(x)\right) d x=0 \quad j=1, . ., q\right\},
$$


where ker $A=\operatorname{Span}\left\{v_{1}, . ., v_{q}\right\}$. Finally, the operator $\mathcal{L}$ considered on the space

$$
\mathbb{R}^{p} \times \mathcal{M}_{n}(\mathbb{R}) \times\left(u_{0}+\widehat{E}\right),
$$

satisfies the assumption of implicit function theorem. This complete the proof of Theorem 3.1. The theorem is proved.

We now give an application of this result to monotone systems for which the properties of operator $A$ are known (see for instance [13]). For that purpose we come back to system (3.5) and we assume that it has a solution $\left(c_{0}, u_{0}\right) \in \mathbb{R} \times\left(C^{2+\alpha}(\mathbb{R})\right)^{n}$ with $u_{0}$ increasing. We assume that:

Assumption 3.3. The matrix $B(x)=f^{\prime}\left(u_{0}(x)\right)$ has nonnegative off-diagonal elements and is irreducible in the functional sense. The matrix $B^{ \pm}=\lim _{x \rightarrow \pm \infty} B(x)$ has a negative principal eigenvalue.

Then we have the following result (see Theorem 5.1 in [13]).

Theorem 3.4. Under Assumption 3.3, the operator A is Fredhom with the zero index. Moreover its kernel is one dimensional and the corresponding eigenfunction $u_{0}^{\prime}(x)$ is positive. Its formally adjoint operator also has a one-dimensional with a positive eigenfunction $v^{*}$.

Finally, as a direct corollary of Theorems 3.1 and 3.4 we obtain the following result:

Theorem 3.5. Assume that system (3.5) has a solution

$$
\left(c_{0}, u_{0}\right) \in \mathbb{R} \times\left(C^{2+\alpha}(\mathbb{R})\right)^{n}
$$

with $u_{0}$ increasing. Assume that assumptions (3.9) and 3.3 are satisfied. Then for some $\epsilon_{0}>0$ and for each $\varepsilon \in \mathcal{M}_{n}(\mathbb{R})$, $\|\varepsilon\|<\epsilon_{0}$ there exists $c(\varepsilon) \in \mathbb{R}$ and $u(\varepsilon) \in\left(C^{2+\alpha}(\mathbb{R})\right)^{n}$ such that

$$
c(0)=c_{0}, \quad u(0)=u_{0},
$$

and $(c(\varepsilon), u((\varepsilon))$ satisfies the problem:

$$
\begin{aligned}
& B u^{\prime \prime}+c u^{\prime}+F(u, \mathcal{F}(\varepsilon, u))=0, \quad x \in \mathbb{R}, \\
& u( \pm \infty)=u^{ \pm} .
\end{aligned}
$$

Proof. Here we have $p=q=1, \tau=c \in \mathbb{R}$ and $M(c)=c I_{n}$. In order to apply Theorem 3.1, it remains to note that

$$
\int_{\mathbb{R}} u_{0}^{\prime}(x) v^{*}(x) d x \neq 0
$$

since $u_{0}^{\prime}>0$ and, due to Theorem $3.4, v^{*}>0$. 


\section{Numerical simulations: competition of species}

In this section we consider the integro-differential system of two equations:

$$
\begin{aligned}
& \frac{\partial u}{\partial t}=d_{1} \frac{\partial^{2} u}{\partial x^{2}}+k_{1} u^{m}\left(1-a_{1} \int_{-\infty}^{\infty} \varphi(x-y) u(y, t) d y-b_{1} \int_{-\infty}^{\infty} \varphi(x-y) v(y, t) d y\right)-p_{1} u, \\
& \frac{\partial v}{\partial t}=d_{2} \frac{\partial^{2} v}{\partial x^{2}}+k_{2} v^{m}\left(1-a_{2} \int_{-\infty}^{\infty} \varphi(x-y) u(y, t) d y-b_{2} \int_{-\infty}^{\infty} \varphi(x-y) v(y, t) d y\right)-p_{2} v .
\end{aligned}
$$

Here $u$ and $v$ are densities of two populations, $m=1$ corresponds to asexual reproduction, $m=2$ to sexual reproduction, the expressions in the brackets describe available resources, $\varphi(x)$ is a piecewise constant function. This system of equations is considered in the interval $0 \leq x \leq L$. The functions $u(x, t)$ and $v(x, t)$ are supposed to be zero for $x \leq 0$ and $x \geq L$. We extend them outside the the interval $[0, L]$ in order to define the integrals in system (4.1), (4.2).

We begin with the case of the co-existence of species where the stationary point $P_{4}$ (see the introduction) is stable. Figure 2 shows the results of numerical simulations for the values of parameters $d_{1}=d_{2}=10^{-5}, k_{1}=k_{2}=0.1, a_{1}=1, b_{1}=0.8, a_{2}=0.8, b_{2}=1, p_{1}=p_{2}=0.007$, $m=1$, the length $L$ of the interval equals 2.5 and the support of the function $\varphi$ is 0.05 . The space interval is divided into 400 space steps shown in the figure. Therefore, the discretization of the space interval is given by the space points $x_{i}, i=0, \ldots, N$, where $x_{0}=0, x_{N}=2.5, N=400$. The initial condition is chosen in such a way that the function $u(x, 0)$ is localized around the point $x_{i}$ with $i=150$ and the function $v(x, 0)$ around $x_{j}$ with $j=250$. They equal zero elsewhere.

Figure 2 shows the space-time evolution of the function $u(x, t)$ (top) and the level lines of both functions $u(x, t)$ and $v(x, t)$ (bottom). There are several waves connecting various stationary points. The function $u(x, t)$ spreads to the left and to the right with respect to the interval where it is initially located. The wave propagating to the left connects the points $P_{0}$ and $P_{1}$. Since $v(x, t)$ is close to 0 in this region, then this wave is close to the wave for the single equation in the monostable case. Its propagation to the right is similar until it meets the wave formed by the function $v(x, t)$. After this moment of time, the species co-exist and the equilibrium $P_{4}$ spreads along the interval.

The behavior described above is similar to that in the local case, that is for the reaction-diffusion system describing competition of species.

We consider next the same values of parameters and the support of the function $\varphi$ two times larger than before. The behavior of the solution is to some extent similar. However, the homogeneous in space solutions lose its stability and periodic in space structures emerge. Therefore, instead of the waves connecting the stationary points we observe waves which connect stationary points with the periodic in space solutions. These are are periodic travelling waves, that is solutions of the form

$$
u(x, t)=U(x-h, t-T), \quad v(x, t)=V(x-h, t-T),
$$

where $h$ and $T$ are some nonzero constants, $T$ is the period. This means that it is a solution which can be obtained from itself by a space shift not for any time as for the usual waves but only for moments of time which differ by the period or its multiples. Periodic travelling waves are well studied for the scalar reaction-diffusion equations with periodic coefficients [2], [3], [11], [14]. The 

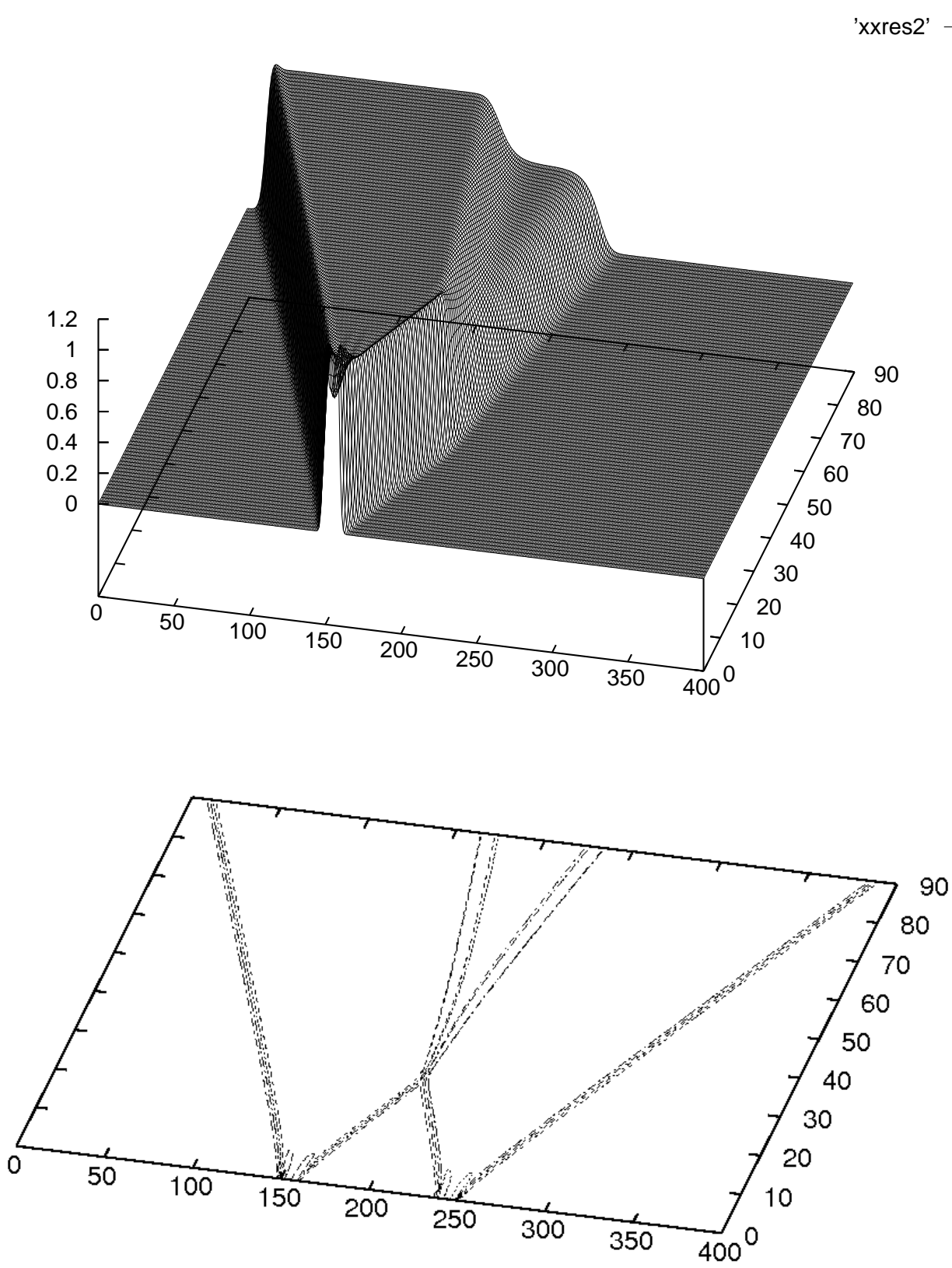

Figure 2: Top: space-time evolution of the function $u(x, t)$. Bottom: level lines of the functions $u(x, t)$ and $v(x, t)$. 
existence of such waves for the integro-differential equations is not proved. They were observed numerically for the single integro-differential equation in [1]. Thus, we observe the propagation of periodic waves, and the periodic in space solution spreads along the space interval. It describes the co-existence of species with a periodic space distribution (Figure 3).

The stationary point $P_{4}$ may not be stable. In this case, the co-existence of species is not generally possible except for the case of a standing wave where the two species exist but are separated in space. If this is not the case, then one of the species dominates and fills gradually the whole space interval while the other species disappears. This corresponds to the travelling wave connecting two stable stationary points $P_{2}$ and $P_{3}$. This behavior is well studied for the reactiondiffusion systems. In the case of integro-differential system where the support of the function $\varphi$ is sufficiently narrow, the existence of waves is proved in Section 3. The results of numerical simulations are shown in Figure 4 for the values of parameters $a_{1}=0.4, b_{1}=1, a_{2}=1, b_{2}=0.8$. It presents the level lines of the functions $u(x, t)$ and $v(x, t)$. The interface separating these two functions in the center of the interval moves to the right signifying that $u(x, t)$ gains space while $v(x, t)$ loses it.

If the support of the function $\varphi$ is larger, then we observe, as before, periodic waves (Figure 5) such that for each $t$ fixed

$$
\begin{aligned}
& u(x, t) \rightarrow 0, x \rightarrow+\infty, \quad u(x, t) \rightarrow u_{0}(x), x \rightarrow-\infty, \\
& v(x, t) \rightarrow 0, x \rightarrow-\infty, v(x, t) \rightarrow v_{0}(x), x \rightarrow+\infty,
\end{aligned}
$$

where $u_{0}(x)$ and $v_{0}(x)$ are some periodic functions. Clearly, the numerical simulations are carried out at a bounded space interval and represent a finite space cut of the periodic wave.

It is interesting to note that average speed of the periodic wave is less than the speed of the usual wave for the same values of parameters (cf. Figures 4 and 5). Some biological interpretations will be discussed below.

Up to now, we have considered the case where the two populations are initially separated, that is the supports of the functions $u(x, 0)$ and $v(x, 0)$ are separated in space. If the initial distributions have the same support or overlapping supports, then the behavior of solutions can change. Figure 6 shows the results of numerical simulations in the case where the support of the function $u(x, 0)$ is the interval of $\mathrm{i}$ from 190 to 210 and the support of $v(x, 0)$ from 185 to 215 . The other values of parameters are the same as in the previous simulations. Though the support of $v(x, 0)$ is larger than of $u(x, 0)$, the values of the parameters determine a faster propagation of $u(x, t)$ which fills a bigger region than the function $v(x, t)$. Figure 6 (top) shows the level lines of the function $u(x, t)$. The function $v(x, t)$ is localized in the inner region (Figure 6, bottom). The interval where the function $v(x, t)$ is localized is slowly growing with time. For other values of parameters it can remain constant.

We finish the description of numerical results with the case of bisexual reproduction $(m=2)$. Figure 7 shows levels lines of the functions $u(x, t)$ and $v(x, t)$ in the case where the supports of the initial conditions are separated in space. The results are qualitatively the same as for $m=1$ (cf. Figure 6): the left part of the interval is filled by the function $u(x, t)$, the right part by the function $v(x, t)$. The interface between them moves slowly to the right. Both, $u(x, t)$ and $v(x, t)$ expand as a periodic travelling wave. The average speed of propagation for $u$ is greater than for $v$. 

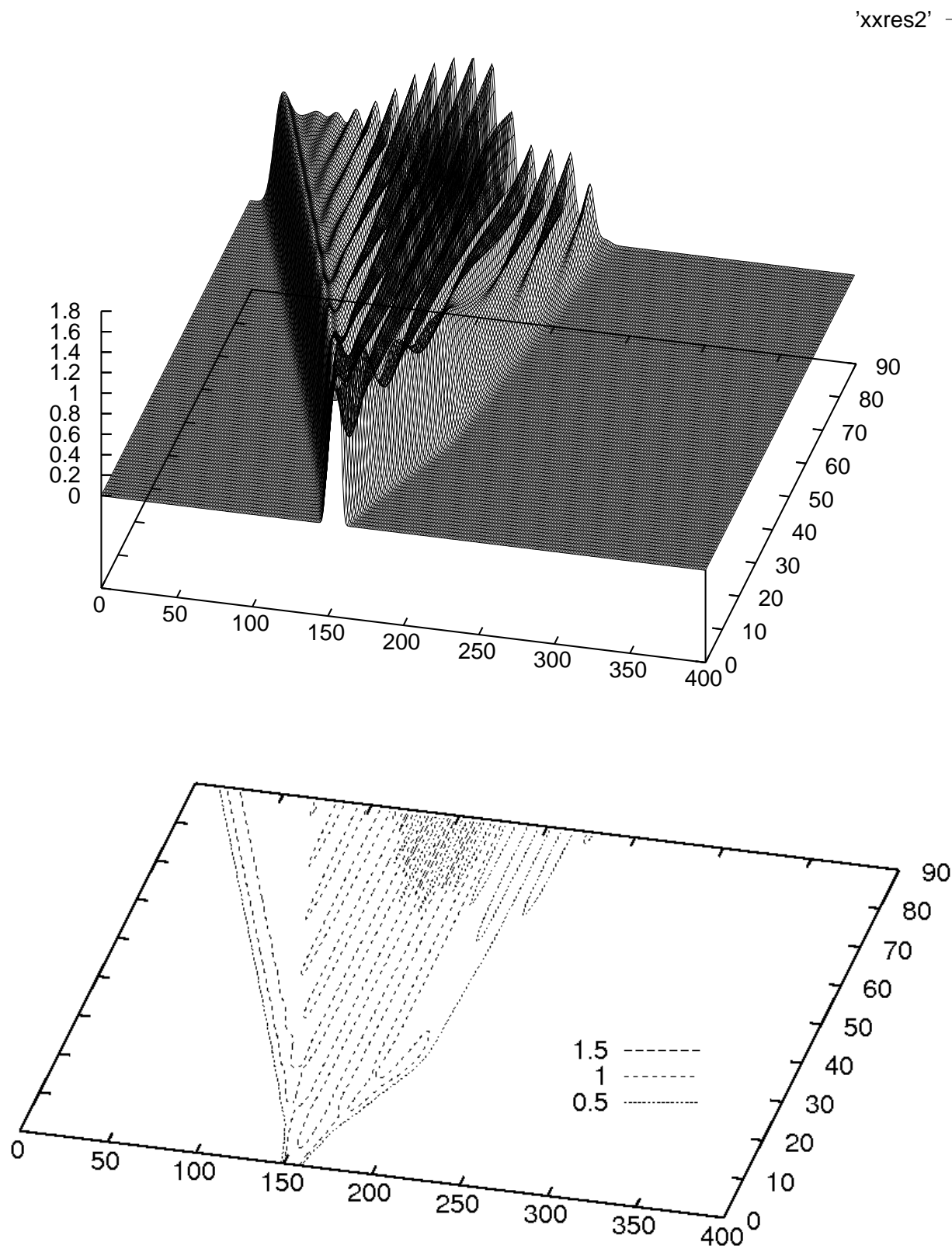

Figure 3: Periodic waves. Top: space-time evolution of the function $u(x, t)$. Bottom: level lines of the functions $u(x, t)$ and $v(x, t)$. 


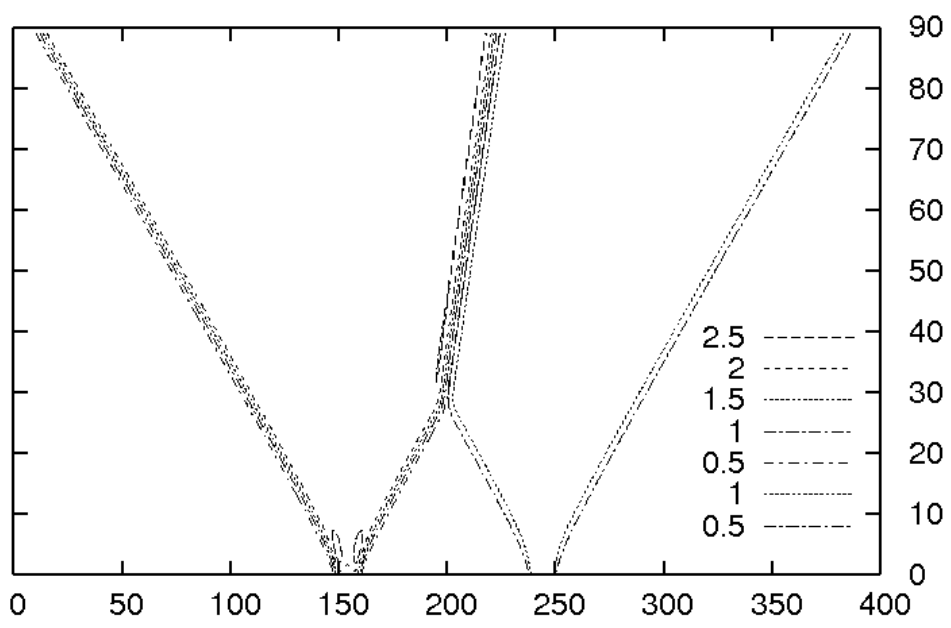

Figure 4: Level lines of the functions $u(x, t)$ and $v(x, t)$ in the case without co-existence.

\section{Discussion}

In this work we study the model of competition of species with nonlocal consumption of resources. In the classical case, competition of species is described by a reaction-diffusion system. In the case of nonlocal consumption of resources it becomes an integro-differential system of equations where the integral terms show that individuals can consume resources in some area around their average location. If the support of the kernel of the integral is sufficiently narrow, then the qualitative behavior of this system is the same as for the reaction-diffusion system. In particular, we prove the existence of travelling waves in the bistable case. The proof is based on the analysis of the linear integro-differential operators: their Fredholm property and solvability conditions. This allows us to apply the implicit function theorem in order to study the nonlinear operators and to show the wave existence.

If the support of the kernel is sufficiently large, then the homogeneous in space solutions can lose their stability and periodic in space stationary solutions emerge. In this case, instead of the usual travelling waves we can observe propagation of periodic waves.

From the biological point of view, nonlocal consumption of resources corresponds to intraspecific competition: individuals of the same species compete for resources. Considered together with random mutation resulting in small changes of the phenotype (diffusion term) and together with the reproduction where descendent have the same phenotype as their parents, intra-specific competition can result in the emergence of new biological species in the process of evolution [6], [7].

In this paper, we are more concerned by the interaction of competition of species with intraspecific competition. Similar to the classical problem of competition of species, two species can co-exist or one of them can disappear while another one will expand. In the latter case, instead of the usual reaction-diffusion wave connecting two stable stationary points (bistable case) we can observe a wave connecting two periodic in space stationary solutions. If the periodic solutions 

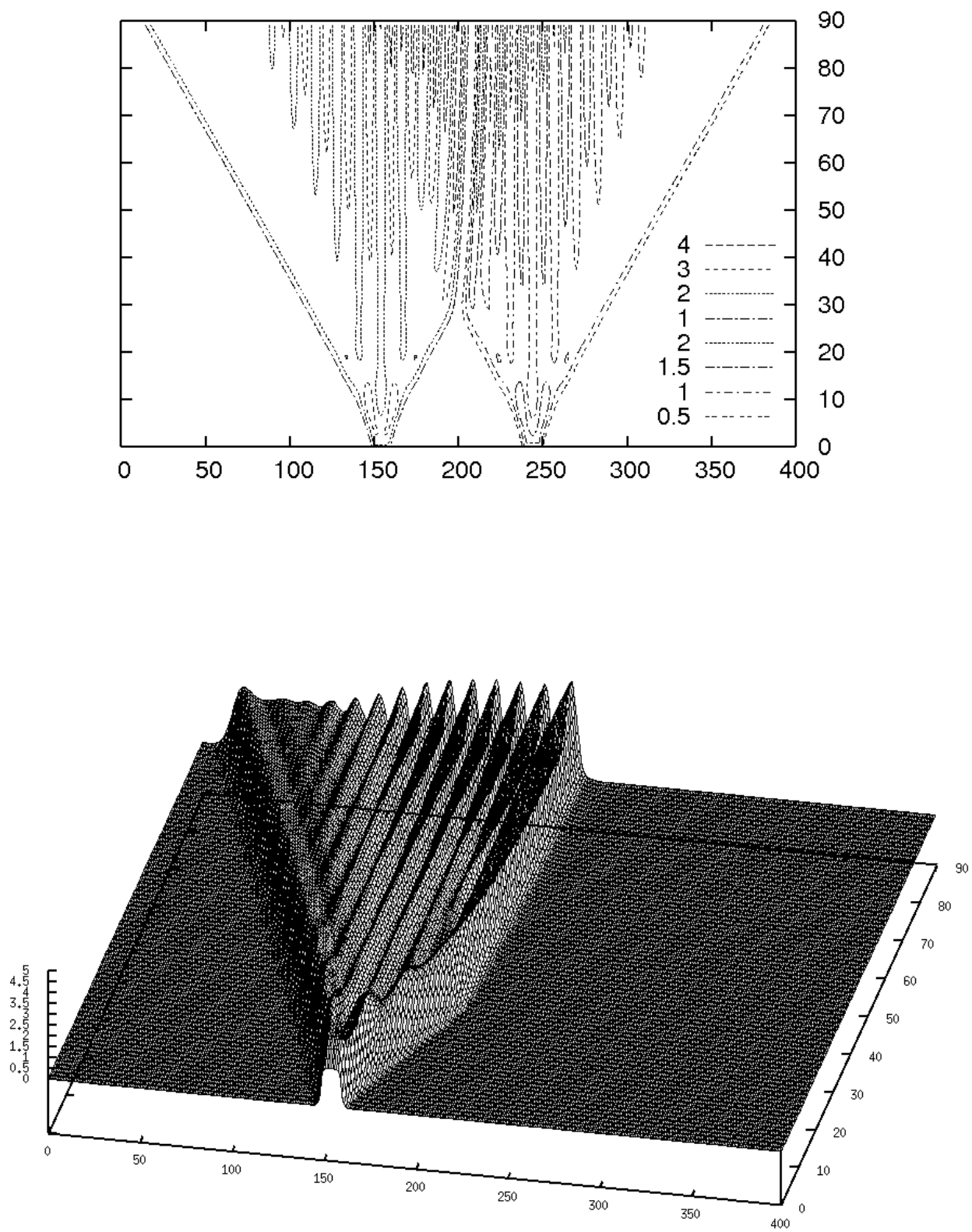

Figure 5: Level lines of the functions $u(x, t)$ and $v(x, t)$ (top) in the case of competition of species; the function $u(x, t)$ (bottom). 

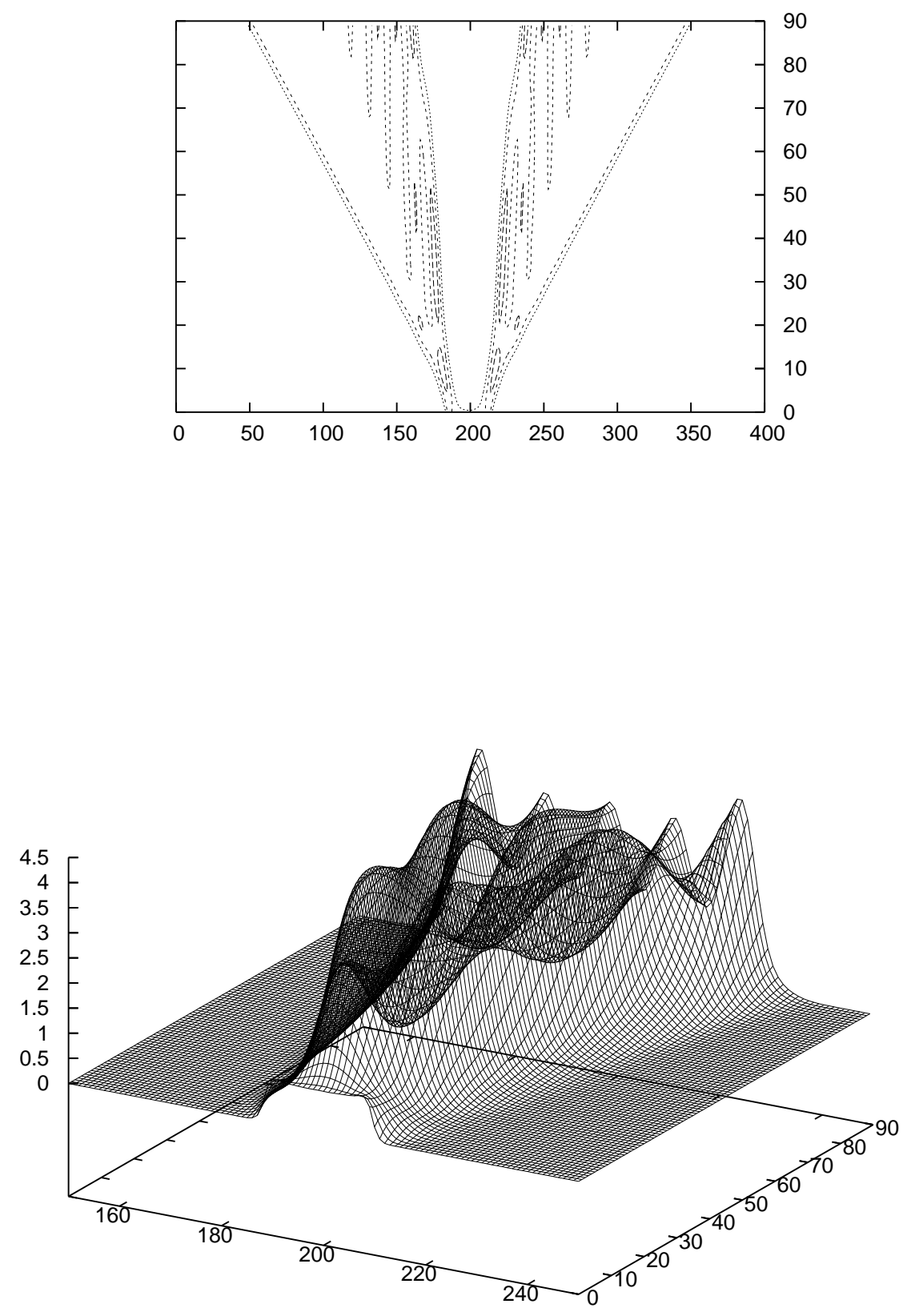

Figure 6: Space-time evolution of the functions $u(x, t)$ and $v(x, t)$ in the case of overlapping initial conditions. 


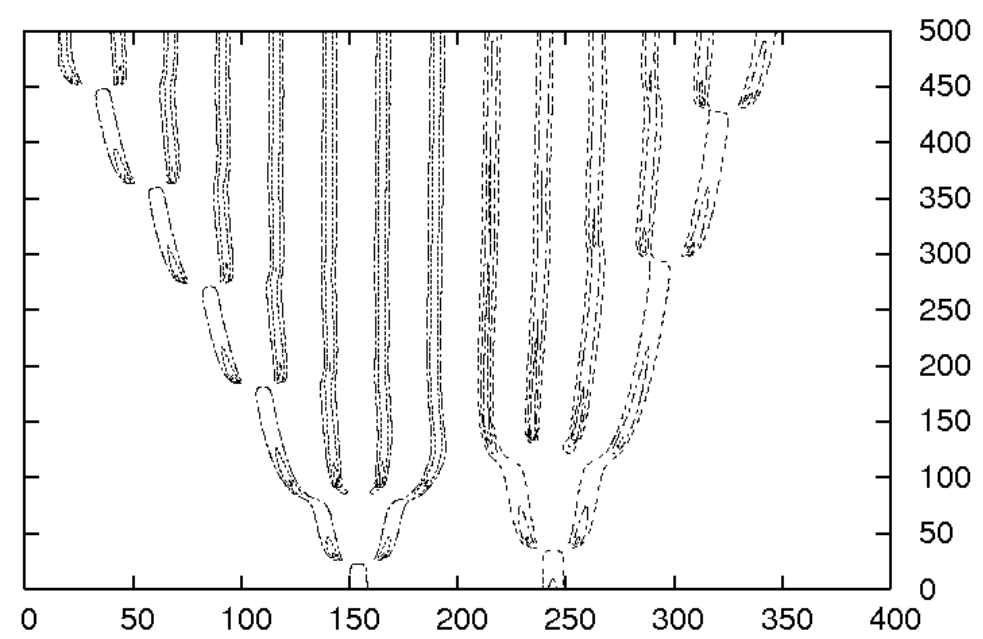

Figure 7: Level lines of the functions $u(x, t)$ and $v(x, t)$ for $m=2$.

have the same period, then the wave can be also periodic. Otherwise it can have a more complex structure.

It is interesting to note that for the same values of parameters, periodic waves have a smaller speed of propagation than the usual waves. This signifies that intra-specific competition helps weaker species to resist to invasion of stronger species.

\section{Acknowledgements}

The work of the first author was supported by the CNCSIS grant, ID 381/2007, Romania.

\section{References}

[1] N. Apreutesei, A. Ducrot and V. A. Volpert, Travelling Waves for Integro-Differential Equations, submitted.

[2] H. Berestycki, F. Hamel. Front propagation in periodic excitable media. Comm. Pure Appl. Math. 55 (2002), No. 8, 949-1032.

[3] H. Berestycki F. Hamel, N. Nadirashvili, The speed of propagation for KPP type problems. I - Periodic framework. J. European Math. Soc., 7 (2005), 173-213.

[4] A. Ducrot, Travelling wave solutions for a scalar age-structured equation, Discrete Contin. Dyn. Syst. Ser. B 7 (2007), no. 2, 251-273 (electronic).

[5] R.A. Fisher. The wave of advance of advantageous genes, Ann.Eugenics, 7 (1937), 355-369. 
[6] S. Genieys, V. Volpert, P. Auger, Pattern and waves for a model in population dynamics with nonlocal consumption of resources, Math. Model. Nat. Phenom. 1 (2006), no. 1, 65-82.

[7] S. Genieys, V. Volpert, P. Auger, Adaptive dynamics: modeling Darwin's divergence principle, Comptes Rendus Biologies, 329 (11), 876-879 (2006).

[8] S. A. Gourley, Travelling front solutions of a nonlocal Fisher equation, J. Math. Biol. 41 (2000), 272-284.

[9] A.N. Kolmogorov, I.G. Petrovsky, and N.S. Piskunov, Étude de l'équation de la diffusion avec croissance de la quantité de matière et son application à un problème biologique, $\mathrm{B}$. Univ. d'Etat à Moscou, Sér. Intern. A 1 (1937), pp.1-26.

[10] O.A. Ladyzhenskaya, N.N. Uraltseva. Linear and quasilinear equations of elliptic type. Moskva, Nauka, 1973.

[11] H. Matano, K.I. Nakamura, B. Lou. Periodic traveling waves in a two-dimensional cylinder with saw-toothed boundary and their homogenization limit. NHN, 4 (2006), 537-568.

[12] A. Volpert, V. Volpert, Elliptic problems with a parameter in unbounded domains, Adv. Differential Equations 12 (2007), no. 5, 573-600.

[13] A. Volpert, Vit. Volpert, Vl. Volpert. Traveling wave solutions of parabolic systems. AMS, Providence, 1994.

[14] J. Xin, Front propagation in heterogeneous media. Siam Rev 42 (2000), 161-230. 